\title{
Can Biorelevant Media be Simplified by using SLS and Tween 80 to Replace Bile Compounds?
}

\author{
Thomas Taupitz and Sandra Klein*
}

\author{
Institute of Pharmaceutical Technology, Johann Wolfgang Goethe University, 9 Max von Laue Street, Frankfurt am \\ Main 60438, Germany
}

\begin{abstract}
In the scientific literature, the use of a surfactant is recommended for both designing quality control tests for water insoluble or sparingly water soluble drugs and for predicting the bioavailability of drugs from various types of formulations. Since the number of poorly soluble drugs is increasing, the selection of adequate dissolution test for these becomes more and more important. The aim of the present study was to develop predictive and discriminatory test methods based on surfactants that are recommended in the literature. Particular respect was given to the use of sodium lauryl sulfate and Tween 80, the two most commonly used surfactants for this purpose. Tamoxifen was used as a model drug. Dissolution experiments were performed using various concentrations of the two surfactants in buffer media typically used to prepare biorelevant test media. Results were then compared with those deriving from the same test formulations in biorelevant and simplified "biorelevant" media. Results from this study indicate that the concentration of surfactant has a huge impact on both the rate and extent of drug release from the formulation and also on the discriminatory power of the test. However, they also indicate that a well designed and validated test medium containing SLS or Tween 80 can be useful in terms of establishing a discriminatory test medium that possibly could also be used to assure batch to batch bioequivalence. Therefore, the approach described in the present paper might be very helpful for developing predictive and discriminatory methods in early formulation development for poorly soluble drugs and which could also be adopted for QC.
\end{abstract}

Keywords: Biorelevant media, surfactants, simplified media, dissolution, poorly soluble drugs.

\section{INTRODUCTION}

Simulation of gastrointestinal conditions is essential to adequately predict the in vivo behavior of poorly soluble drugs. Thus, simulating small intestinal conditions with biorelevant media such as fasted state simulated intestinal fluid (FaSSIF) and fed state simulated intestinal fluid (FeSSIF) has become standard practice in many dissolution laboratories [1-6]. However, due to their complex composition, these media are expensive and their stability is limited. Therefore, in a precursor study [7] selected media containing synthetic surfactants with physicochemical properties similar to those of FaSSIF and FeSSIF were identified. Such simplified "biorelevant" media can be used to replace biorelevant test media for screening formulations, if it can be shown that solubility and dissolution of the drug in these simplified media are similar to that in real biorelevant media prepared with bile components. But, the cited experiments have also shown that validation of the correspondence of results in media containing synthetic surfactants with those containing bile components is necessary on a case-by-case basis. They also indicated that various types and concentrations of surfactants can result in very different dissolution profiles.

*Address correspondence to this author at the Institute of Pharmaceutical Technology, Johann Wolfgang Goethe University, 9 Max von Laue Street, Frankfurt am Main 60438, Germany; Tel: ++49 (0)69-798-29679 / -29689; Fax: ++49 (0)69-798-29694; E-mail: Sandra.Klein@em.uni-frankfurt.de
In the scientific literature, the use of a surfactant is recommended for both designing quality control (QC) tests for water insoluble or sparingly water soluble drugs and for predicting the bioavailability of drugs from various types of formulations [8]. However, most of these references do not give any instructions on how to develop discriminative dissolution methods based on media containing surfactants and, they do not take into account the various physicochemical parameters that were taken into account when the simplified „biorelevant“ media were established [7]. Thus, particularly for inexperienced analysts or at time points when in vivo data are not yet available the selection of adequate test conditions can represent a great challenge, where the choice of an inadequate model could result in both under- or overprediction of the in vivo dissolution behavior. Such a model might also lack of the discriminative power in terms of distinguishing between "good" and "bad" batches of a given formulation.

Thus, in the present series of tests results from real biorelevant media, i.e. FaSSIF and FeSSIF [1] and simplified "biorelevant" media having physicochemical properties that correspond to those of FaSSIF and FeSSIF [7] were compared with those generated in media containing various concentrations of surfactants that are typically recommended in the literature [9-11]. Particular respect was given to two of the most commonly used surfactants, sodium lauryl sulfate (SLS) and Tween 80. 


\section{MATERIALS AND METHODS}

\section{Materials}

Tween 80 - Crillet $^{\circledR} 4$ (polyoxyethylene sorbitan monooleate), lot \# 0000333555 (Croda Europe Ltd., East Yorkshire, England) and SLS (sodium lauryl (dodecyl) sulfate), lot \# L56042192709 (VWR International Ltd., Poole, England) were of analytical grade and purchased commercially.

Tamoxifen citrate (lot \# SRP 01106t) was obtained from Sequoia Research Products Ltd., Pangbourne, UK. Tamoxifen Hexal $20 \mathrm{mg}$, lot \#61MR19 (HEXAL AG, Holzkirchen, Germany) was purchased commercially. White opaque gelatin capsules (Wepa Nr. 35758 according to DAB 10 ; size 1; $0.5 \mathrm{~mL}$ ) were purchased from Wepa Apothekenbedarf $\mathrm{GmbH} \& \mathrm{Co} \mathrm{KG}$, Hillscheid, Germany. All other compounds were of analytical grade and purchased commercially.

\section{Media Preparation}

First, Blank FaSSIF pH 6.5 and Blank FeSSIF pH 5.0 (i.e. the relevant buffers for the preparation of biorelevant media representing small intestinal conditions in the fasted or fed state) were prepared as follows: Blank FaSSIF was prepared by dissolving $3.438 \mathrm{~g} \mathrm{NaH}_{2} \mathrm{PO}_{4}$ and $6.186 \mathrm{~g}$ of $\mathrm{NaCl}$ in $900 \mathrm{~mL}$ of demineralized water, adjusting the $\mathrm{pH}$ to 6.5 by addition of $0.1 \mathrm{~N} \mathrm{NaOH}$ solution and finally adjusting the volume to $1000.0 \mathrm{~mL}$ with demineralized water. Correspondingly, Blank FeSSIF was prepared by dissolving $11.874 \mathrm{~g} \mathrm{NaCl}$ in $900 \mathrm{~mL}$ of demineralized water, adding $8.65 \mathrm{~g}$ of glacial acetic acid, adjusting the $\mathrm{pH}$ to 5.0 with 0.1 $\mathrm{N} \mathrm{NaOH}$ solution and bringing the volume to $1000.0 \mathrm{~mL}$ by addition of demineralized water. Then simplified media containing SLS and Tween 80 were prepared by adding the various concentrations, i.e. $0.1,0.175,0.25,0.5,0.75,1.0$ and $1.5 \%$ of the surfactants to each of the two buffers. Subsequently, the media were placed in an ultrasonic bath for 15 min and finally stirred for another $15 \mathrm{~min}$ on a magnetic stirrer before use.

\section{Selection of Model Drug Formulations and Test Parame- ters}

Since its oral bioavailability and dissolution rate performance has been reported to depend on the presence of dissolution enhancers $[12,13]$, tamoxifen was selected as a model drug for the experiments. Tamoxifen is a BCS class II drug [14], meaning that its bioavailability is mainly determined by the rate and extent of tamoxifen dissolution. Results from preliminary experiments have also shown that drug release from various tamoxifen formulations is sensitive to the composition of the test media. For these reasons, the drug appeared to be an optimal candidate to prove the applicability of simplified media on the basis of SLS and Tween 80.

The intention of the first series of tests was to find concentrations of SLS and Tween 80 which create tamoxifen dissolution profiles homomorphous to those obtained with FaSSIF, FeSSIF and simplified "biorelevant" media. These initial screening experiments were performed with a formulation that in the precursor study did not exhibit complete drug release, but about $50 \%$ dissolution in within the test duration. This formulation consisted of gelatin capsules, filled with $1 / 2$ tablet Tamoxifen Hexal $20 \mathrm{mg}$ and $15.17 \mathrm{mg}$ of tamoxifen citrate. The resulting test dose was therefore 30.34 $\mathrm{mg}$ tamoxifen citrate $(=20 \mathrm{mg}$ tamoxifen $)$ in all cases The formulations were subject to dissolution studies in Blank FaSSIF and Blank FeSSIF containing SLS or Tween 80 in the concentration range mentioned above. For comparison purposes, drug release of the intact Tamoxifen Hexal $20 \mathrm{mg}$ tablet was also recorded in Blank FaSSIF and Blank FeSSIF. Results from these experiments were then compared with those obtained with the corresponding formulation in simplified "biorelevant" FaSSIF and FeSSIF in the precursor study [7]. Finally, the surfactant concentrations that resulted in dissolution profiles similar to those in the precursor study were selected for further experiments.

The focus of the second part of the study was to elucidate the discriminatory power of the selected SLS and Tween 80 media. For this purpose, a set of "good" and "bad" batches of a tamoxifen citrate formulation was generated. Similar to the precursor study, neither different batches of a formulation nor in vivo data were available at the time of the experiment. The latter situation displays a typical moment in early formulation development when prototypes for pilot bioavailability studies need to be selected. For the above mentioned reasons and to better compare current results with those obtained with simplified "biorelevant" media [7], the compromise was to perform the release experiments with different qualities of a "self-made" capsule formulation containing mixtures of different ratios of the poorly soluble pure drug and partial tablets of the same drug.

\section{Dissolution Studies}

Drug release experiments were performed with USP Apparatus 2 (Paddle, DT 600 and DT 700, Erweka, Germany). Each vessel was filled with $500 \mathrm{~mL}$ of medium, and an agitation speed of $75 \mathrm{rpm}$ was used for all dissolution studies [15]. Experiments were run in triplicate. Samples $(5 \mathrm{~mL})$ were removed after 5, 10, 15, 20, 30, 45, 60, 90, and $120 \mathrm{~min}$ using a glass syringe and, immediately filtered through a $0.45 \mu \mathrm{m}$ Teflon $^{\circledR}$ filter (FP 030/2, Whatman, Schleicher \& Schuell GmbH, Dassel, Germany). To keep the test volume constant throughout the entire test, the sample volume was replace by fresh preheated $\left(37^{\circ} \mathrm{C}\right)$ medium.

\section{UV Analysis}

All samples were analyzed at $275 \mathrm{~nm}$ using a UVspectrophotometer (U 2000/3000, Hitachi Ltd, Tokyo, Japan) equipped with a $5 \mathrm{~mm}$ cuvette. A separate standard calibration curve was prepared in each of the media tested.

\section{RESULTS AND DISCUSSION}

Figs. (1 and 2) display a copy of the results obtained in the precursor study where, as mentioned before, the "selfmade" tamoxifen formulations represented very good candidates for examining the discriminatory power of simplified "biorelevant" test media. In Blank FaSSIF and Blank FeSSIF, the Tamoxifen Hexal $20 \mathrm{mg}$ tablets showed a poor dissolution performance, i.e. in Blank FaSSIF only $10 \%$ of drug stayed in solution within the test duration of $120 \mathrm{~min}$ and in Blank FeSSIF about $35 \%$ of the drug was released. However, in biorelevant and simplified "biorelevant" test media, the tablet formulation showed almost complete drug 


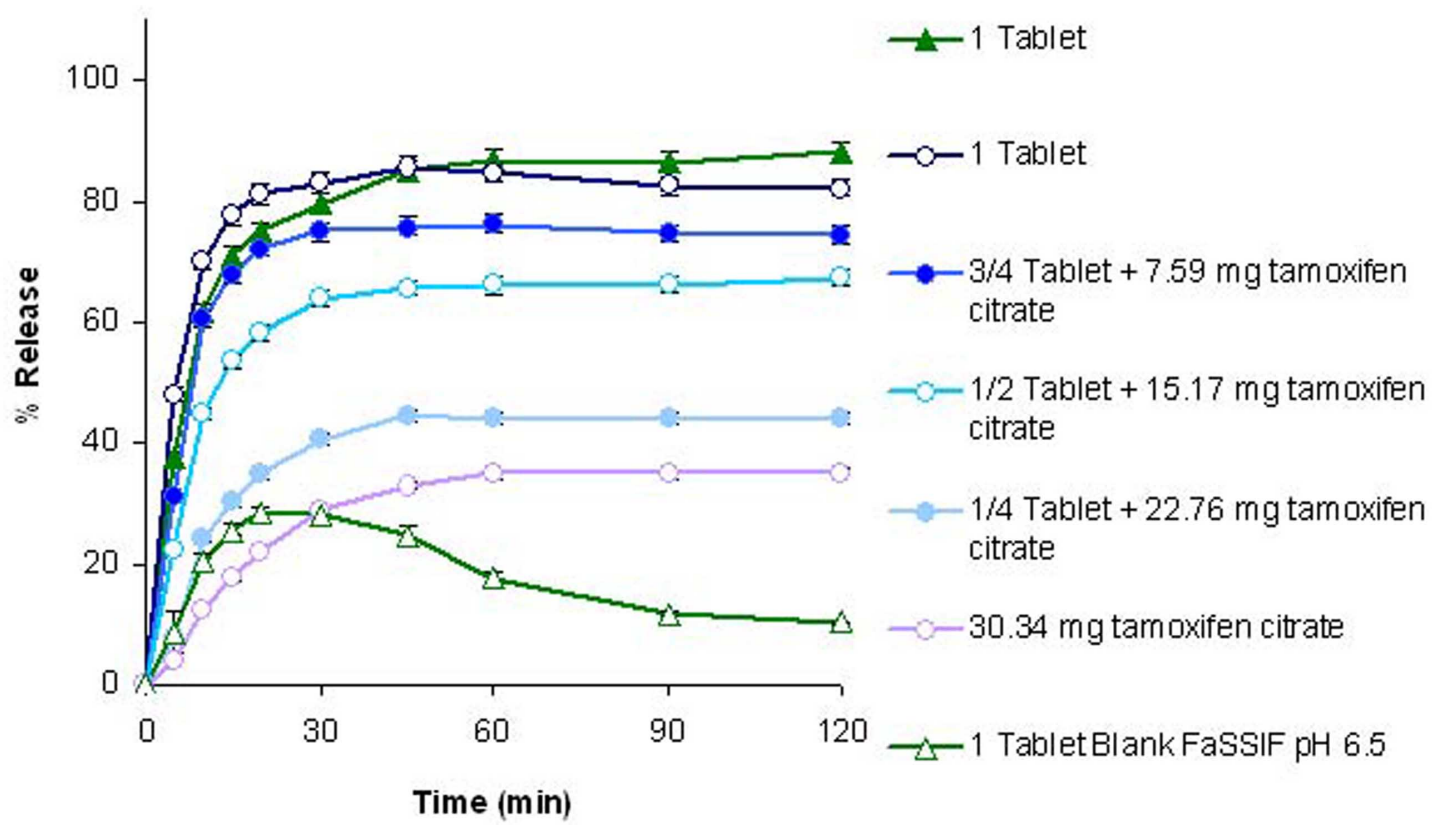

Fig. (1). Drug release of Tamoxifen Hexal $20 \mathrm{mg}$, Tamoxifen Hexal/tamoxifen citrate mixtures, and tamoxifen citrate in media simulating the fasted state: $\triangle$ Blank FaSSIF, $\boldsymbol{\Delta}$ FaSSIF, $\bigcirc \& \bullet$ : simplified "biorelevant" FaSSIF (copied with permission from Dissolution Technologies 2007, 14 (4): p. 8-13).

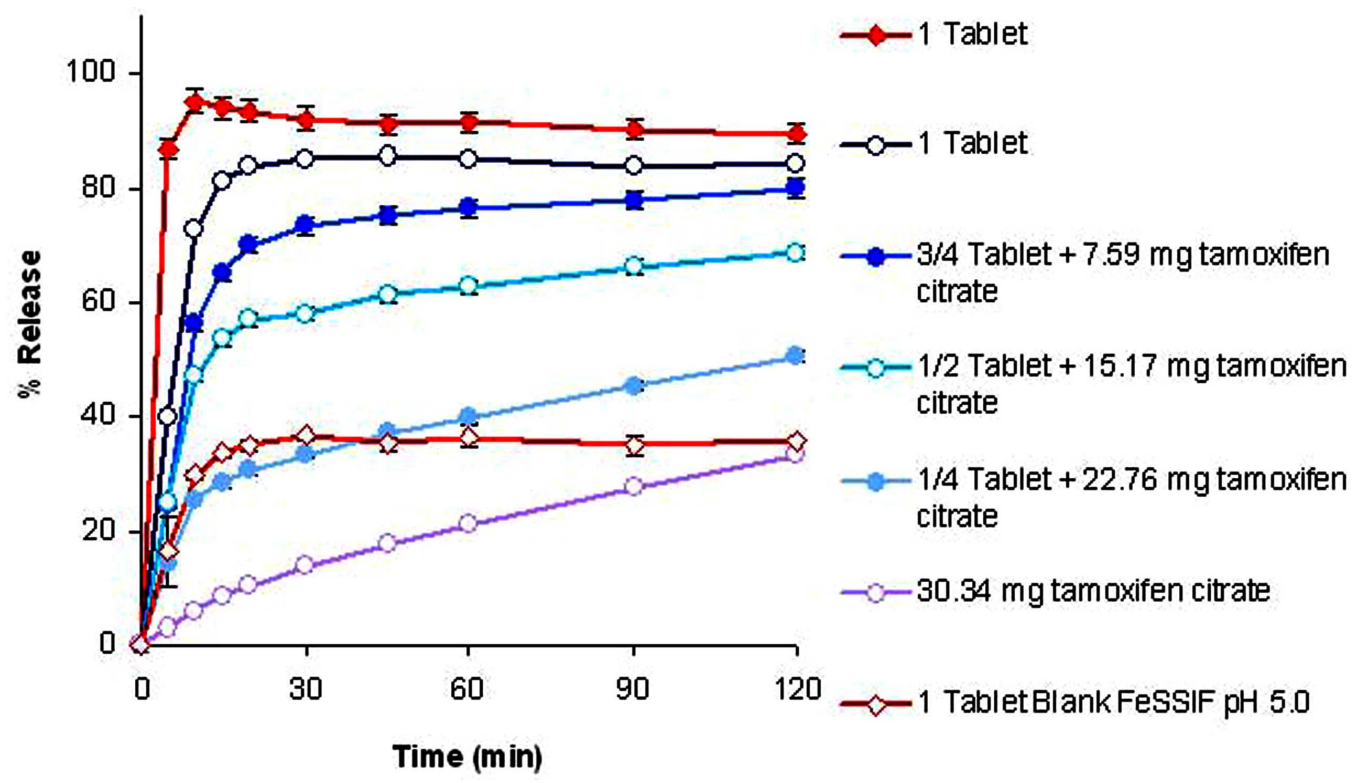

Fig. (2). Drug release of Tamoxifen Hexal $20 \mathrm{mg}$, Tamoxifen Hexal/tamoxifen citrate mixtures, and tamoxifen citrate in media simulating the fed state: $\diamond$ Blank FeSSIF, $\bullet$ FeSSIF, $\bigcirc \& \bullet$ : simplified "biorelevant" FeSSIF (copied with permission from Dissolution Technologies 2007, 14 (4): p. 8-13).

release. The pure drug in contrast showed a relatively poor dissolution behavior in both biorelevant and simplified "biorelevant" test media $(\leq 35 \%$ drug release within 120 min). Due to the significantly different dissolution performances of the tablet formulation and the pure drug, dissolution profiles of the different "self-made" test formulations could be clearly rank-ordered in terms of the ratio of their ingredients.

The objective of the present series of tests was first to find SLS- and Tween 80 based media that result in dissolution profiles that are superimposable to those obtained with the simplified "biorelevant" media. Thus, in the first set of experiments gelatin capsules filled with $1 / 2$ tablet Tamoxifen Hexal $20 \mathrm{mg}$ and $15.17 \mathrm{mg}$ of tamoxifen citrate were examined in Blank FaSSIF and Blank FeSSIF containing SLS or Tween 80 in the concentration range of 0.1-1.5\%.

Results of these experiments (data not shown here) indicated that dissolution profiles in media containing $0.1-0.5 \%$ SLS and 0.1-0.25\% Tween 80 come very close to the target profiles. Concentrations of $0.1 \%$ SLS and $0.175 \%$ Tween 80 were selected as the lowest applicable concentrations that can be used to resemble dissolution profiles obtained in 
FaSSIF/FeSSIF and the simplified "biorelevant" media established in the precursor study (see results for $1 / 2$ tablet Tamoxifen Hexal $20 \mathrm{mg}$ and $15.17 \mathrm{mg}$ of tamoxifen citrate in Figs. 1 and 2).

As another intention of the study was to design test media having a discriminatory power comparable to that of the "simplified" biorelevant media, in the next set of experiments, the selected media were systemically screened for their discriminatory power, i.e. they were used to screen the dissolution behavior of different qualities of a "self-made" capsule formulation. The results of these experiments are shown in Figs. (3-6).
Even if the same concentration of SLS or Tween 80 was used to simulate both, the fasted and the fed state, results obtained with the new set of media came close to that obtained with the physiologically based media. This was particularly the case for the simplified FaSSIF media. Figs. (3 to 6) clearly indicate that the new media have an adequate discriminatory power. Like with the simplified "biorelevant" media, in all cases it was possible to distinguish between the different compositions of the formulations tested. However, it needs to be mentioned that even if the profiles in the different media came close to each other, some profiles showed marked differences in the rate and extent of initial drug re-

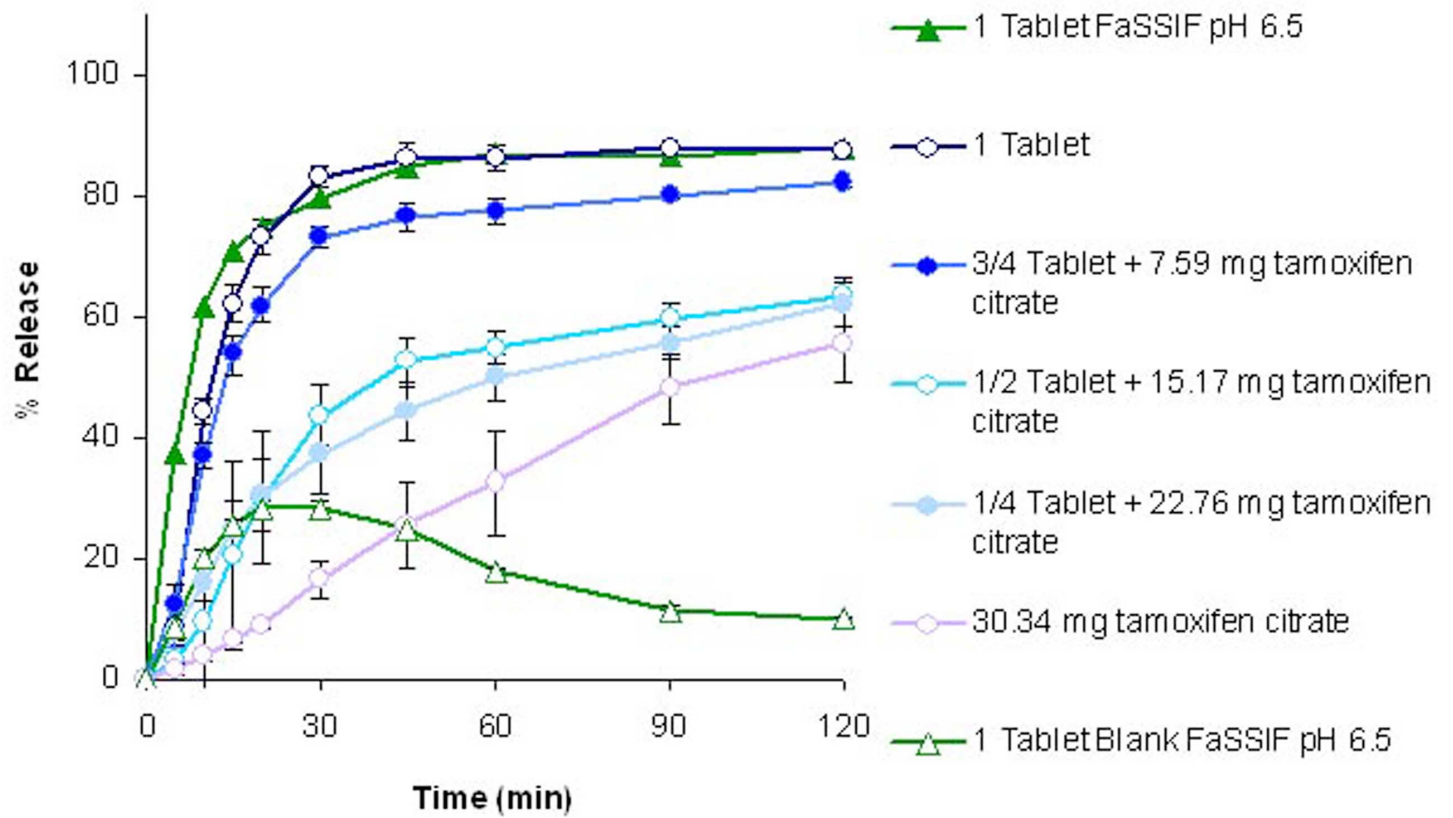

Fig. (3). Drug release of Tamoxifen Hexal $20 \mathrm{mg}$, Tamoxifen Hexal/tamoxifen citrate mixtures, and tamoxifen citrate in media simulating the fasted state: $\triangle$ Blank FaSSIF, $\boldsymbol{\Delta}$ FaSSIF, $\bigcirc \& \bullet$ : Blank FaSSIF with $0.1 \%$ SLS.

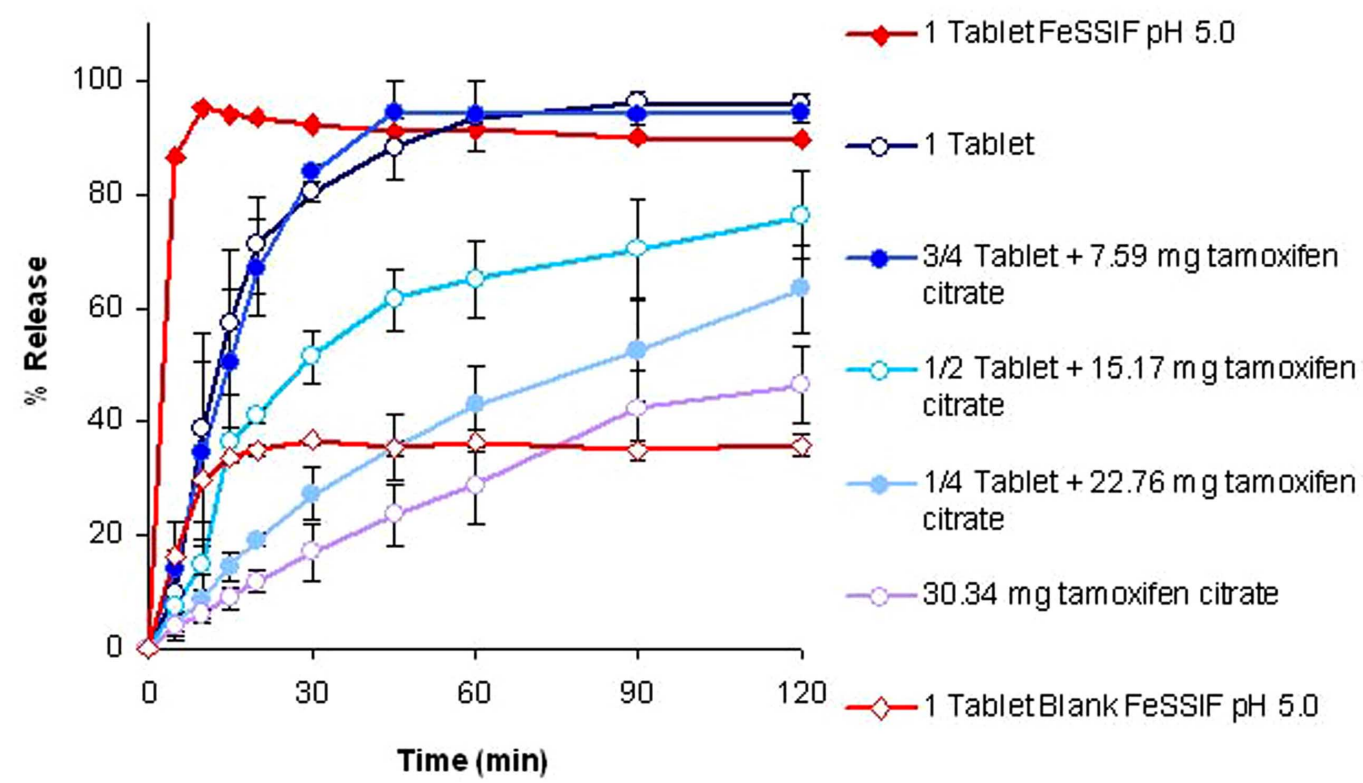

Fig. (4). Drug release of Tamoxifen Hexal $20 \mathrm{mg}$, Tamoxifen Hexal/tamoxifen citrate mixtures, and tamoxifen citrate in media simulating the fed state: $\diamond$ Blank FeSSIF, $\bullet$ FeSSIF, $O \& \bullet:$ Blank FeSSIF with $0.1 \%$ SLS. 


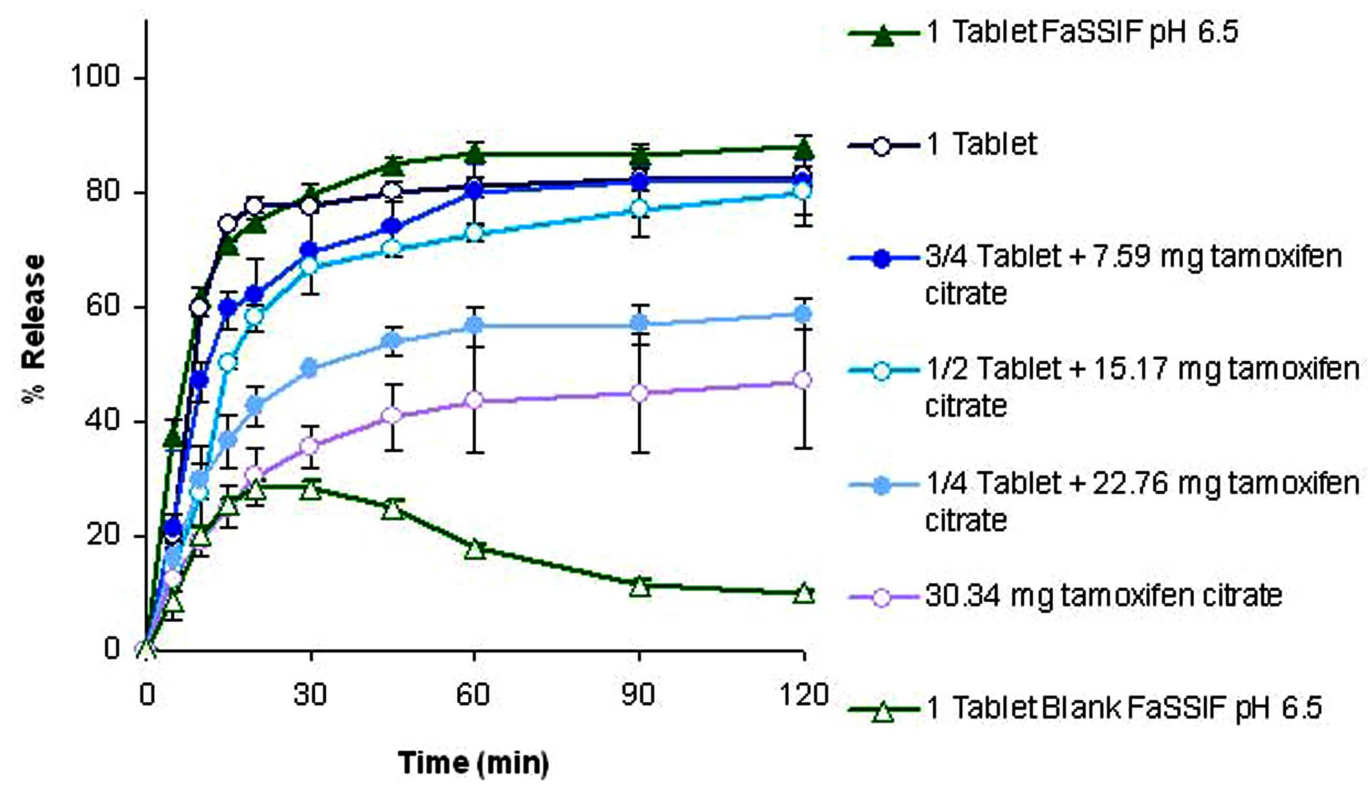

Fig. (5). Drug release of Tamoxifen Hexal $20 \mathrm{mg}$, Tamoxifen Hexal/tamoxifen citrate mixtures, and tamoxifen citrate in media simulating the fasted state: $\triangle$ Blank FaSSIF, $\Delta$ FaSSIF, $\bigcirc \& \bullet$ : Blank FaSSIF with $0.175 \%$ Tween 80.

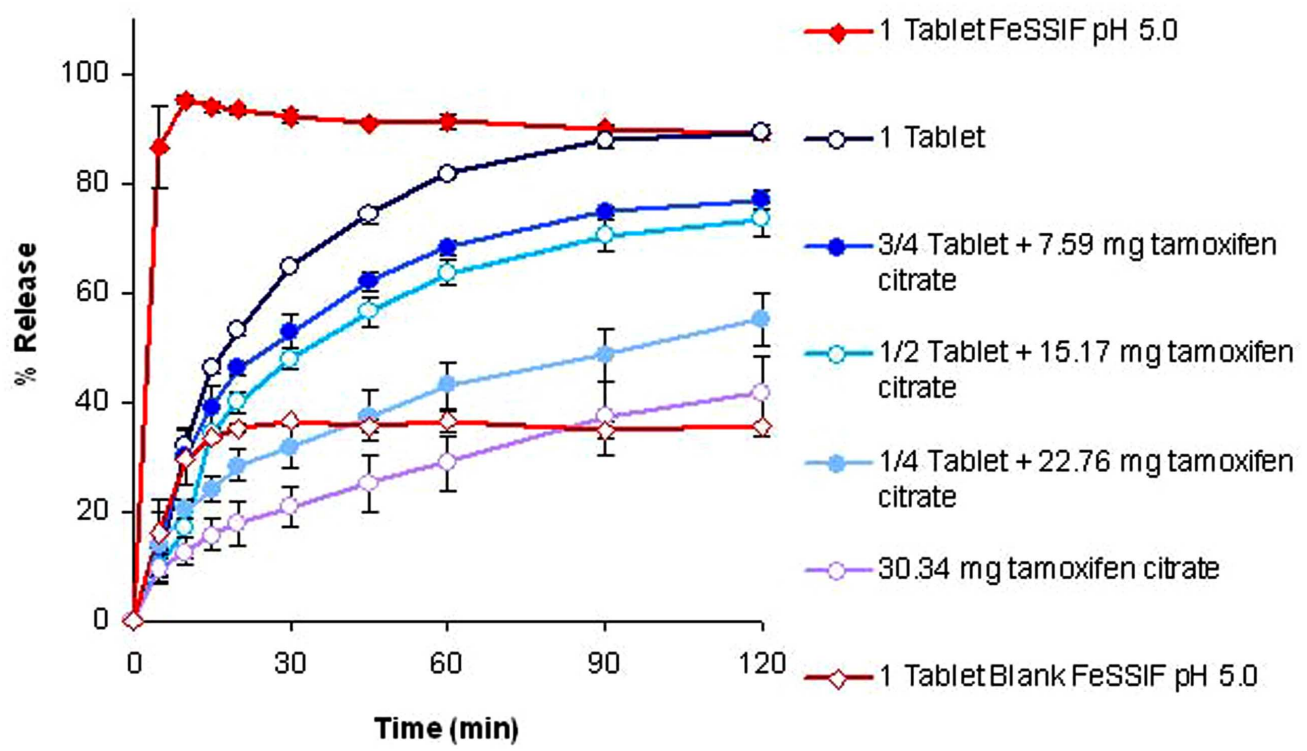

Fig. (6). Drug release of Tamoxifen Hexal $20 \mathrm{mg}$, Tamoxifen Hexal/tamoxifen citrate mixtures, and tamoxifen citrate in media simulating the fed state: $\diamond$ Blank FeSSIF, $\diamond$ FeSSIF, $O \& \bullet$ : Blank FeSSIF with $0.175 \%$ Tween 80.

lease from the formulations. Overall, as already observed in the precursor study, with the simplified media it was much more difficult to simulate drug release in FeSSIF. It could be noted that in simulated FeSSIF containing $0.175 \%$ Tween 80 (see Fig. 6) the initial drug release rate was smaller than in simplified "biorelevant" FeSSIF and original FeSSIF. Nevertheless, the amount of drug released after a total test time of 120 min was again similar to that in corresponding reference media. As can be seen in Figs. (7-10), similarity to the results in simplified "biorelevant" media was definitely no longer guaranteed when the same set of experiments was performed with media containing higher concentrations of the two surfactants.
Figs. (7-10) show the dissolution profiles of the test formulations in media containing $1 \%$ SLS or Tween 80. Comparison of these dissolution profiles with those shown in Figs. (3-6) clearly indicates that not only the type of surfactant but also the choice of the surfactant concentration has a huge impact on both the rate and extent of in vitro drug release and also the discriminatory power of the test method. As discussed before, the use of the media containing $0.1 \%$ SLS and $0.175 \%$ Tween 80 resulted in an excellent discriminatory power of the test system. Although the use of higher concentrations of the two surfactants, i.e. $1 \%$ SLS and $1 \%$ Tween 80 , enabled to achieve a better simulation of the initial release rate in some of the experiments, these con- 


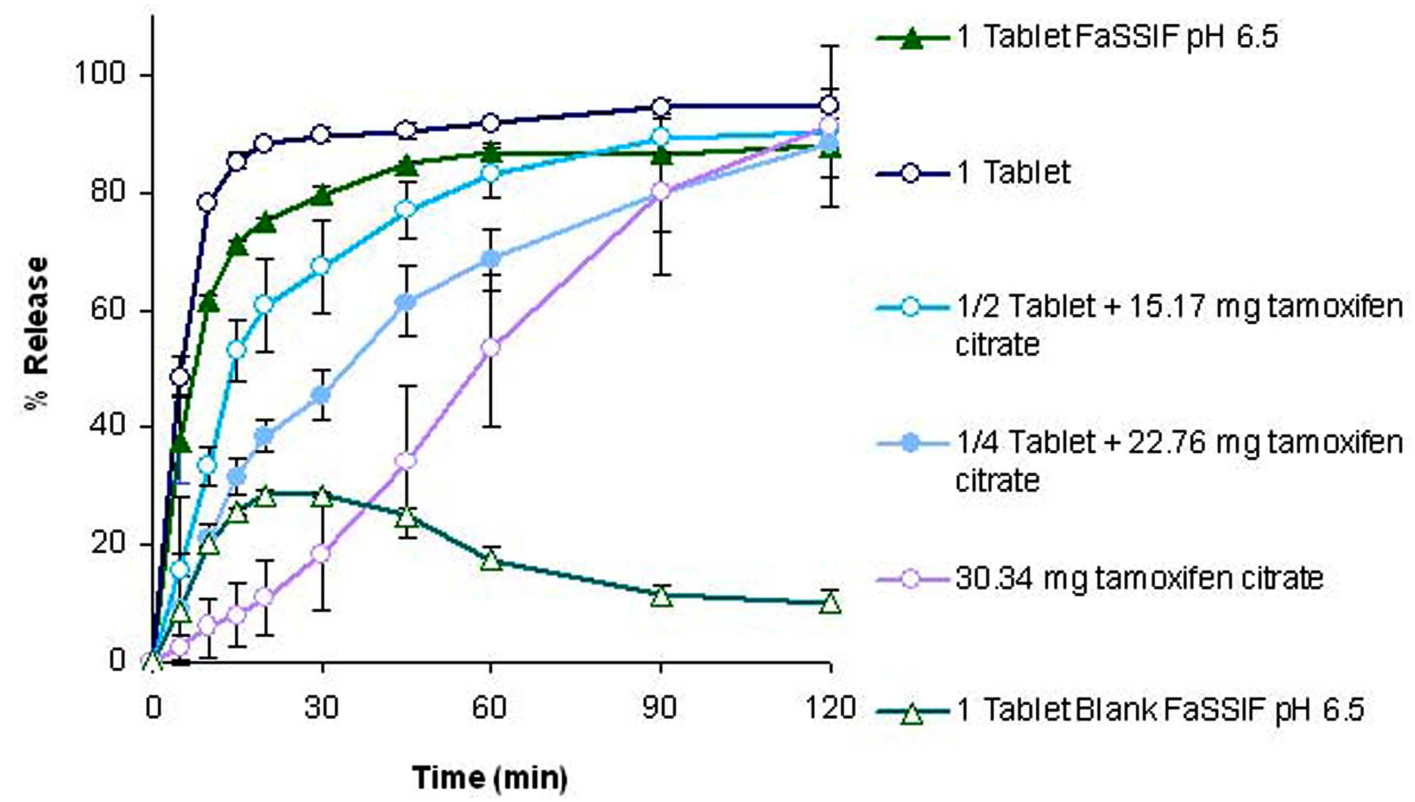

Fig. (7). Drug release of Tamoxifen Hexal $20 \mathrm{mg}$, Tamoxifen Hexal/tamoxifen citrate mixtures, and tamoxifen citrate in media simulating the fasted state: $\triangle$ Blank FaSSIF, $\boldsymbol{\Delta}$ FaSSIF, $O \& \bullet$ : Blank FaSSIF with $1 \%$ SLS.

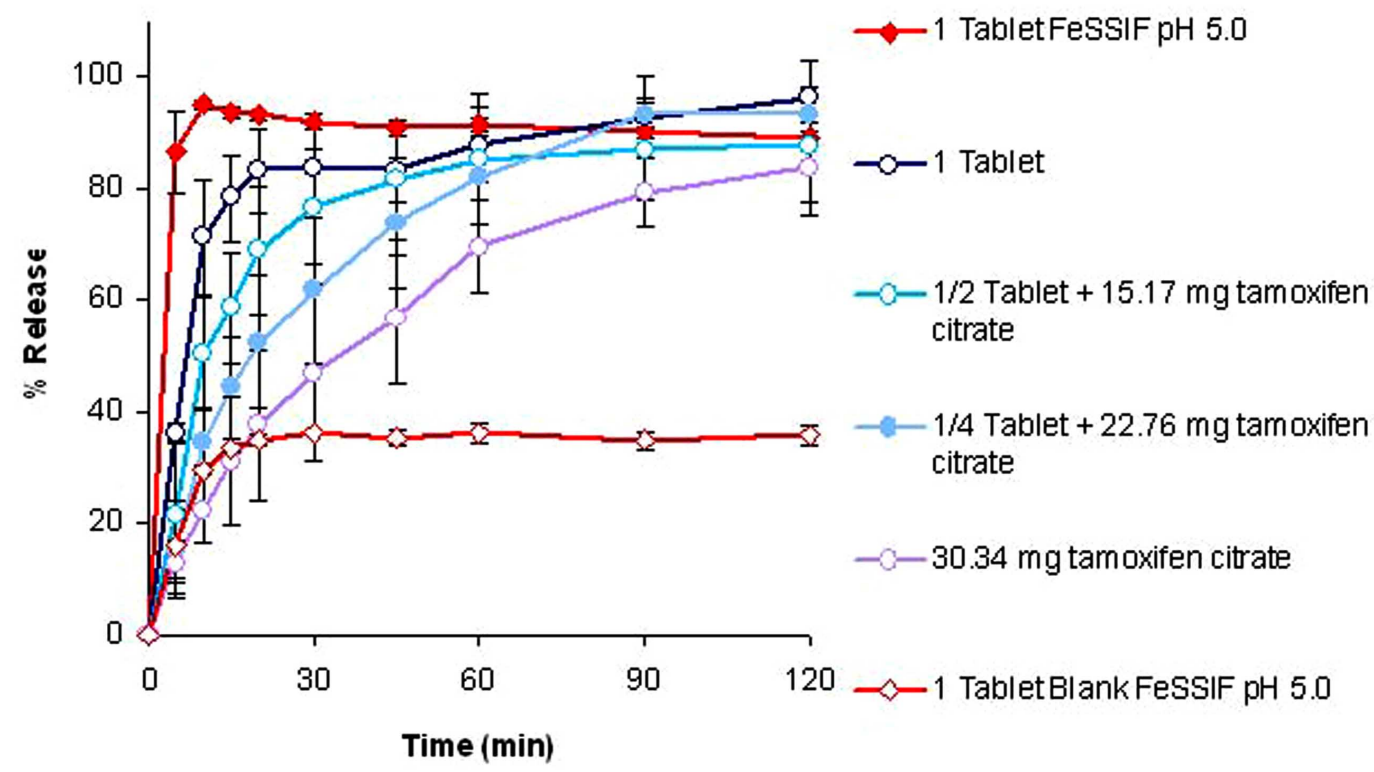

Fig. (8). Drug release of Tamoxifen Hexal $20 \mathrm{mg}$, Tamoxifen Hexal/tamoxifen citrate mixtures, and tamoxifen citrate in media simulating the fed state: $\diamond$ Blank FeSSIF, $\bullet$ FeSSIF, $\bigcirc \& \bullet::$ Blank FeSSIF with $1 \%$ SLS.

centrations turned out to be unfeasible in terms of discriminating between the different test formulations. After the total test time of $120 \mathrm{~min}$ in the media containing $1 \%$ of surfactant nearly the same amount of drug was released from all test formulations and even the pure drug did completely dissolved in this time frame. This implies a very poor discriminatory power of this test setup. This observation was also supported by the standard deviations calculated for the average drug release from triplicate measurements. For all profiles in media containing $1 \%$ of surfactant the standard deviations were significantly higher than those obtained in media containing $0.1 \%$ SLS and $0.175 \%$ Tween 80 , respectively.
It can be summarized that based on the results obtained in the present study, commonly used surfactants like SLS and Tween 80 represent excipients that can be very useful in the development of discriminatory dissolution tests for formulations containing poorly soluble compounds. However, results from the present study also indicate the importance of screening for the right medium composition. Even if methods applying up to $5 \%$ SLS and up to $2 \%$ Tween 80 are described in international pharmacopoeia and guidances [9, 11], such methods should not be simply adapted to other drug products. The results presented here suggest that an uncritical use of methods described in the literature is likely to come along with test methods that will be anything but 


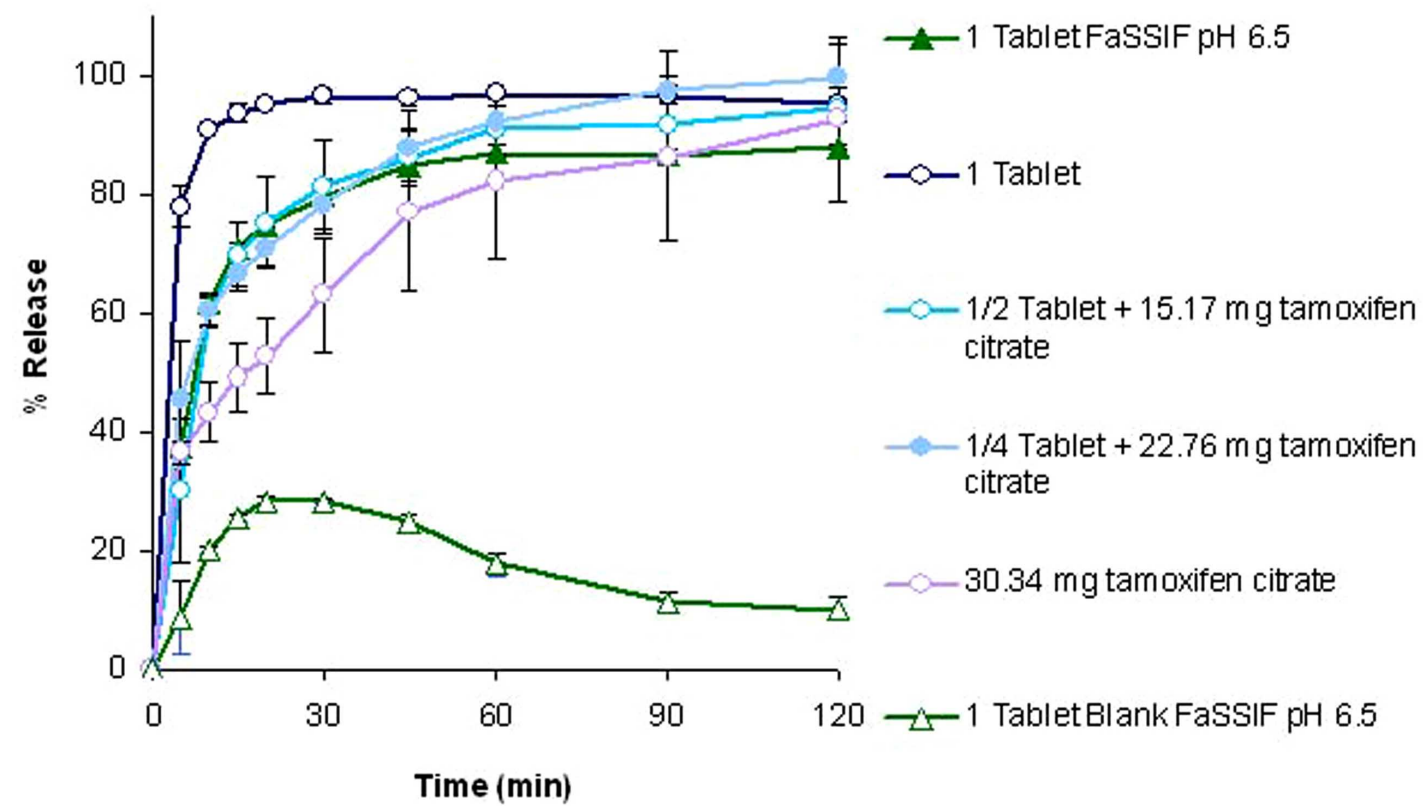

Fig. (9). Drug release of Tamoxifen Hexal $20 \mathrm{mg}$, Tamoxifen Hexal/tamoxifen citrate mixtures, and tamoxifen citrate in media simulating the fasted state: $\Delta$ FaSSIF, O: Blank FaSSIF with $1 \%$ Tween 80.

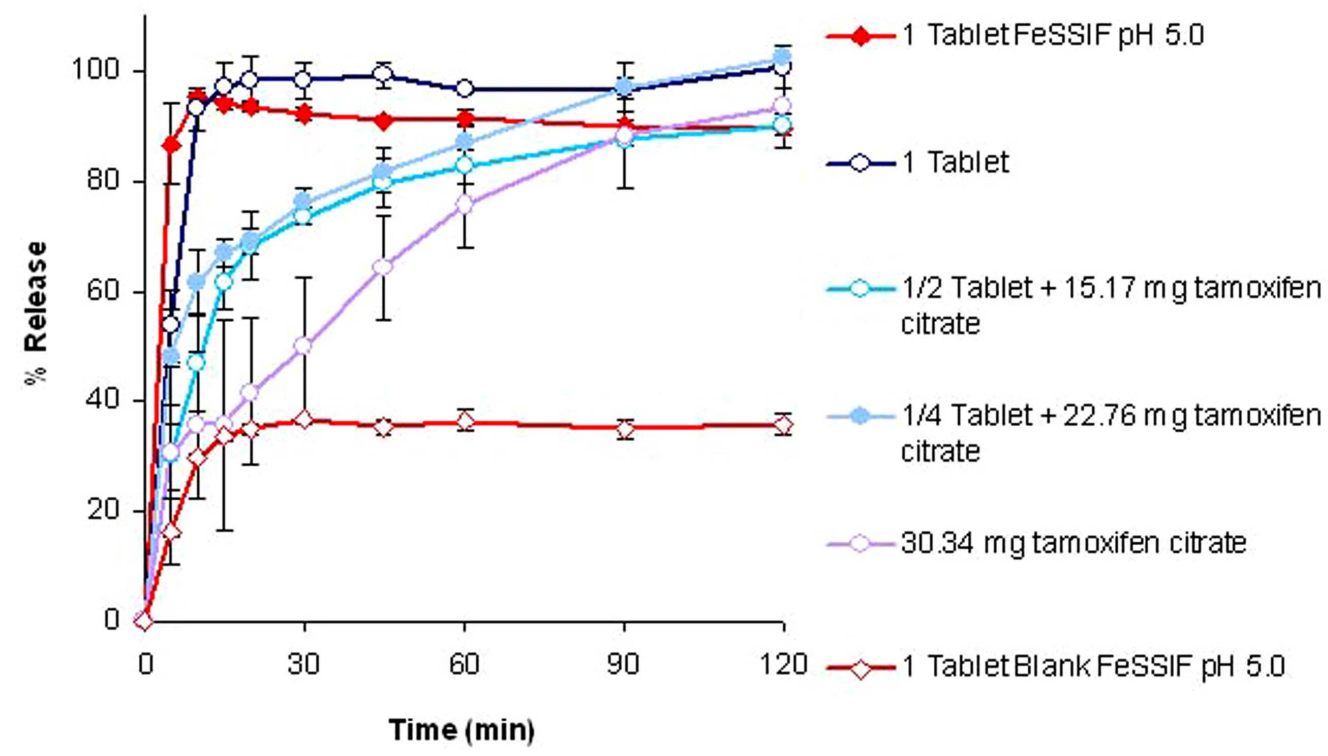

Fig. (10). Drug release of Tamoxifen Hexal $20 \mathrm{mg}$, Tamoxifen Hexal/tamoxifen citrate mixtures, and tamoxifen citrate in media simulating the fed state: $\checkmark$ FeSSIF, O: Blank FeSSIF with $1 \%$ Tween 80.

predictive for in vivo drug release and with respect to QC purposes will most likely also lack of discriminatory power in terms of assuring batch to batch bioequivalence for new drug products.

\section{CONCLUSION}

The approach described in the present paper can help to develop drug release tests which, even if based on the use of non-physiological surfactants and without taking into account all relevant physicochemical parameters at the sites of drug release and absorption, might feature a certain grade of predictive power for the in vivo drug performance of new drug formulations. Although the validation of such test systems will require some time, all in all this procedure will be of great benefit for the user since for routine analysis well designed simplified media come along with various advantages which include the discriminatory power, the in vivo predictivity and, particularly when compared with the use of physiological surfactants, the time- and cost efficiency. Thus, provided it can be shown that solubility and dissolution of the drug and some selected preformulations in these simplified media are similar to that in biorelevant media prepared with bile components, simplified media can be very useful in the course of both screening various prototypes and developing quality control tests of a given drug formulation. 


\section{REFERENCES}

[1] Galia, E.; Nicolaides, E.; Horter, D.; Lobenberg, R.; Reppas, C.; Dressman, J. B. Evaluation of various dissolution media for predicting in vivo performance of class I and II drugs. Pharm. Res., 1998, 15(5), 698-705.

[2] Nicolaides, E.; Galia, E.; Efthymiopoulos, C.; Dressman, J. B.; Reppas, C. Forecasting the in vivo performance of four low solubility drugs from their in vitro dissolution data [In Process Citation]. Pharm. Res., 1999, 16(12), 1876-1882.

[3] Dressman, J. B.; Reppas, C. In vitro-in vivo correlations for lipophilic, poorly water-soluble drugs. Eur. J. Pharm. Sci., 2000, 11 (Suppl 2), 73-80.

[4] Kostewicz, E. S.; Brauns, U.; Becker, R.; Dressman, J. B. Forecasting the oral absorption behavior of poorly soluble weak bases using solubility and dissolution studies in biorelevant media. Pharm. Res., 2002, 19(3), 345-349.

[5] Nicolaides, E.; Hempenstall, J.; Reppas, C. Biorelevant dissolution tests with flow-through apparatus. Dissolution Technol., 2000, 7(1), 8-11.

[6] Nicolaides, E.; Symillides, M.; Dressman, J. B.; Reppas, C. Biorelevant dissolution testing to predict the plasma profile of lipophilic drugs after oral administration. Pharm. Res., 2001, 18(3), 380-388.

[7] Zoeller, T.; Klein, S. Simplified biorelevant media for screening dissolution performance of poorly soluble. Drugs Dissolution Technol., 2007, 14(4), 8-13.

[8] EMEA, Note for Guidance on the Investigation of Bioavailability and Bioequivalence. Committee for Proprietary Medicinal
Products (CPMP), The European Agency for the Evaluation of Medicinal Products - Human Medicines Evaluation Unit: London, 2000 .

[9] USP 32; United States Pharmacopoeia Convention, Inc.: Rockville MD, 2009.

[10] FDA, Guidance for Industry: Dissolution Testing of Immediate Release Solid Oral Dosage Forms. U.S. Department of Health and Human Services, Food and Drug Administration, Center for Drug Evaluation and Research (CDER): Rockville MD, USA, 1997.

[11] FDA, FDA-Recommended Dissolution Methods Database. In CDER. http://www.accessdata.fda.gov/scripts/cder/dissolution [accessed October 22, 2009].

[12] Buchanan, C. M.; Buchanan, N. L.; Edgar, K. J.; Lambert, J. L.; Posey-Dowty, J. D.; Ramsey, M. G.; Wempe, M. F., Solubilization and dissolution of tamoxifen-hydroxybutenyl cyclodextrin complexes. J. Pharm. Sci. 2006, 95(10), 2246-2255.

[13] Buchanan, C. M.; Buchanan, N. L.; Edgar, K. J.; Little, J. L.; Malcolm, M. O.; Ruble, K. M.; Wacher, V. J.; Wempe, M. F. Pharmacokinetics of tamoxifen after intravenous and oral dosing of tamoxifen-hydroxybutenyl-beta-cyclodextrin formulations. J. Pharm. Sci., 2007, 96(3), 644-660.

[14] Wu, C. Y.; Benet, L. Z. Predicting drug disposition via application of BCS: Transport/absorption/elimination interplay and development of a biopharmaceutics drug disposition classification system. Pharm. Res., 2005, 22(1), 11-23.

[15] WHO Working document QAS/04.109/Rev. 1. Proposal to waive in vivo bioequivalence requirements for the WHO model list of Essential medicines immediate release, solid oral dosage forms. In Working Document. World Health Organisation: Geneva, 2005.

(C) Taupitz and Klein; Licensee Bentham Open.

This is an open access article licensed under the terms of the Creative Commons Attribution Non-Commercial License (http://creativecommons.org/licenses/by-nc/3.0/) which permits unrestricted, non-commercial use, distribution and reproduction in any medium, provided the work is properly cited. 